\title{
Quantum Materials for Energy-Efficient Computing
}

\author{
SUGATA CHOWDHURY, ${ }^{1,2,3,7}$ HOULONG ZHUANG, ${ }^{3,8}$ \\ SHAWN COLEMAN (1) ${ }^{3,4,9}$ SRIKANTH PATALA, ${ }^{3,5,10}$ and JACOB BAIR ${ }^{3,6,11}$
}

\begin{abstract}
1.--National Institute of Standards and Technology, Gaithersburg, MD 20899, USA. 2.-Department of Physics and Astronomy, Howard University, Washington, DC 20059, USA. 3.- School for Engineering of Matter, Transport and Energy, Arizona State University, Tempe, AZ 85287, USA. 4.-U.S. Army Research Laboratory, Aberdeen Proving Ground, MD 21005, USA. 5.-Department of Materials Science and Engineering, North Carolina State University, Raleigh, NC 27695, USA. 6.-Department of Mechanical Engineering, Brigham Young University, Provo, UT, USA. 7.-e-mail: sugata.chowdhury@nist.gov. 8.—e-mail: hzhuang7@asu.edu. 9.—e-mail: shawn.p. coleman8.civ@mail.mil. 10._e-mail: spatala@ncsu.edu. 11.—e-mail: jacob.bair@pnnl.gov
\end{abstract}

Recent advancements in experimental tools, computational methods, computing power, and materials informatics present us with an exciting opportunity to predictively discover and design materials for a variety of technologically relevant applications. In particular, quantum mechanical $\mathrm{ab}$ initio methods such as density functional theory, dynamical mean-field theory, quantum Monte-Carlo simulations, and time-dependent density functional theory have been playing a pivotal role in developing a fundamental microscopic understanding of complex phenomena, and in the discovery and design of a number of quantum materials for energy applications.

The current topic, "Quantum Materials for Energy-Efficient Computing," offer JOM-readers with an update on recent progress in this field. All the included papers on this topic have been invited. They cover the state-of-the-art in the application as well as the integration of computational methods, particularly ab initio simulation methods, with experiments and materials informatics applied to the discovery and design of emerging materials.

Multiporphyrin supramoleculars are very interesting and versatile classes of $\pi$-conjugated systems and very useful for optical switches, conductive materials, and nonlinear optics. One of the unsolved problems related to these materials is the effect of

Sugata Chowdhury, Houlong Zhuang, Shawn Coleman, and Jacob Bair are JOM Advisors, and Srikanth Patala is guest editor for this topic, which is sponsored by the Computational Materials Science and Engineering Committee of TMS.

(Received July 14, 2020; accepted July 14, 2020) the end-group molecules on the different optical properties and applications. The first paper, "SpinPolarized Transport and Optoelectronic Properties of a Novel-Designed Architecture with a PorphyrinBased Wheel and Organometallic Multidecker Sandwich Complex-Based Axle" by Gao et al., discusses the architecture of the novel "wheel-andaxle" molecule $(c-\mathrm{P} 6)_{m} /(\mathrm{FeBz})_{n}$, with $(c-\mathrm{P} 6)$. In this article, the authors investigate the electronic structure, transport property, and linear photoresponse of the supramolecule. The results indicate that (c$\mathrm{P} 6) \mathrm{m} /(\mathrm{FeBz}) \mathrm{n}$ is an excellent candidate for spinfilters, and exhibits a distinct polarized photoresponse character. Additionally, the authors demonstrate that $(\mathrm{c}-\mathrm{P} 6) \mathrm{m} /(\mathrm{FeBz}) \mathrm{n}$ is a very good candidate for an optical switch, because it can operate within a considerable photon energy range. All these novel properties make (c-P6)m/(FeBz)n an attractive material for electronic and optoelectronic devices.

Several emerging materials, such as superconductors, topological insulators, magnetic materials, photocatalysts, battery materials, and quantum materials, exhibit promising quantum properties, like quantum fluctuations, quantum coherence, quantum entanglement, and electron-electron interactions. In the second paper, entitled "Modified Failure Mechanism of Silicon through Excess Electrons and Holes", Shen and An investigate the electron-hole interaction in quantum materials. In this work, the authors considered silicon ( $\mathrm{Si}$ ) as the prototype material and employed density functional theory to investigate how electrons and holes influence its deformation and mechanical properties. They demonstrate that, electrons and holes weaken the bonds in single-crystal $\mathrm{Si}$ and nanotwinned $\mathrm{Si}$, and therefore plastically deform under shear strain, whereas the neutral state system experiences a structure recovery during shear 
deformation. Additionally, the bonds along twin boundaries break due to excess electrons and holes. This article provides insights into the role of both excess electrons and holes in modifying the mechanical properties of Si.

Over the last two decades, the scientific and engineering communities have increased the focus on alternative energy materials because current fossil fuels have a devastating impact on our climate. With the continuous increase in universal energy demand and the desire to combat climate change, our last paper focuses on the effect of the quantum materials for lithium-ion batteries (LIBs). The title of the article is "Graphene Nanoplatelet Additives for High C-rate LiFePO4 Battery Cathodes" and is by Adepoju et al. The authors introduced graphene nanoplatelets (GNPs) as conductive additives at the cathodes of lithiumion phosphate $\left(\mathrm{LiFePO}_{4}\right)$ batteries and study their effect on battery performance, specifically under high current rates. They found that the GNPs increase the flexibility in the three-dimensional conductive network and the discharge capabilities. The authors mentioned that the battery performance is enhanced due to the GNP bridge and the larger surface area. These findings indicate that quantum materials can enhance the current rate at the cathodes of LIBs.

The following papers are being published in the September issue, under the topic of Quantum Materials for Energy-Efficient Computing:

1. "Spin-Polarized Transport and Optoelectronic Properties of a Novel-Designed Architecture with a Porphyrin-Based Wheel and Organometallic Multidecker Sandwich Complex-Based Axle" by Gao et al. https://link.springer.com/article/10.10 07/s11837-020-04153-0

2. "Modified Failure Mechanism of Silicon through Excess Electrons and Holes" by Shen and An. http s://link.springer.com/article/10.1007\%2Fs11837-02 0-04180-x

3. "Graphene Nanoplatelet Additives for High Crate $\mathrm{LiFePO} 4$ Battery Cathodes" by Adepoju et al. https://link.springer.com/article/10.1007/s 11837-020-04224-2

Publisher's Note Springer Nature remains neutral with regard to jurisdictional claims in published maps and institutional affiliations. 